\title{
Effectiveness of Bailing capsules in the treatment of lupus nephritis: A meta-analysis
}

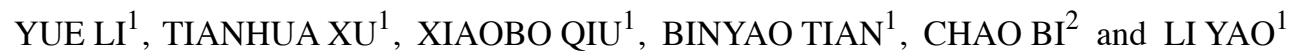 \\ ${ }^{1}$ Department of Nephrology, The First Hospital of China Medical University, Shenyang, Liaoning 110001; \\ ${ }^{2}$ Zhejiang University School of Medicine, Hangzhou, Zhejiang 310029, P.R. China
}

Received October 24, 2019; Accepted April 4, 2020

DOI: $10.3892 / \mathrm{mmr} .2020 .11293$

\begin{abstract}
Previous studies have explored the treatment of lupus nephritis with Bailing capsules; however, due to limited sample sizes and inconsistent results across these studies, no definitive conclusions have been drawn. Thus, the present study aimed to provide evidence for the effectiveness of Bailing capsules in the treatment of lupus nephritis. To obtain relevant clinical studies (published before 20 July 2019), PubMed, Embase, Cochrane Library, China National Knowledge Infrastructure, WanFang and the Chinese Biomedical Literature Database were searched, and relevant studies concerning the use of Bailing capsules for treating lupus nephritis were selected. The extracted data were general characteristics such as the first author, publication year, study year, follow-up time, age, sex, course of the disease and a number of outcome indicators. These included systemic lupus erythematosus disease activity index (SLEDAI) score, serum albumin (Alb), 24-h urinary protein, serum creatinine, anti-ds-DNAIgM, complement component 3 (C3), and the number of effective treatments and complications. Meta-analysis was performed using R-3.12 software. Publication bias was assessed using Egger's test. A total of 14 studies comprising 1,301 participants were combined for analysis in the present study. The results demonstrated that with the exception of anti-ds-DNAIgM and complement C3, other indicators, such as SLEDAI score, Alb, 24-h urinary protein, serum creatinine, and the number of effective treatments and complications) in the Bailing capsule treatment group were improved compared with those in the control group. The results of the present meta-analysis suggested that Bailing capsules may be effective in the treatment of lupus nephritis.
\end{abstract}

Correspondence to: Dr Li Yao, Department of Nephrology, The First Hospital of China Medical University, 155 Nanjing North Street, Shenyang, Liaoning 110001, P.R. China

E-mail: yaoli1680@163.com

Key words: lupus nephritis, systemic lupus erythematosus, Bailing capsule, meta-analysis

\section{Introduction}

Lupus nephritis is a kidney inflammation caused by systemic lupus erythematosus (SLE) (1). SLE is characterized by persistent and severe inflammation that damages multiple organs (2). In particular, $60-80 \%$ of patients with SLE develop renal or urinary function abnormalities (3). It has been estimated that in North America, the annual prevalence of SLE is 23.2/100,000 and 241/100,000 for males and female, respectively (4). In addition, $>50 \%$ of patients with SLE have lupus nephritis (5); thus, it is necessary to find effective methods for the treatment of lupus nephritis.

The main treatment regimen for lupus nephritis are immunosuppressants, such as corticosteroids and cyclophosphamide $(6,7)$; however, the use of immunosuppressants increases the risk of infections in patients (8). In view of the limitations of immunosuppressants, the use of Bailing capsules, which are prepared from the dry powder of Ophiocordyceps sinensis mycelium, has been explored previously in lupus nephritis therapy (9). Bailing capsules possess antihypoxic, anti-inflammatory and antitumor effects, regulate the endocrine system and enhance the immune function (10). Bailing capsules have been widely used in adjuvant therapy of glomerulonephritis, pyelonephritis, nephrotic syndrome and other diseases without adverse effects (11). Studies have reported the treatment of lupus nephritis with Bailing capsules. For example, Zhou et al (12) indicated that treatment with Bailing capsules regulated cellular immunity levels in patients with lupus nephritis. A previous study in patients with lupus nephritis indicated that the 24-h urinary protein, serum creatinine and urea nitrogen levels after treatment were markedly lower in the treatment group (Bailing capsules with cyclophosphamide) compared with those in the control group (13). In a similar study, the serum creatinine, 24-h urinary protein, $\beta_{2}$ microglobulin and SLE Disease Activity Index (SLEDAI) scores in the treatment group (Bailing capsules + Leflunomide + Prednisone acetate) were lower compared with those in the control group, whereas the albumin (Alb), complement C3 and red blood cell (RBC) levels were higher compared with those of the control group (14). Despite the existence of multiple studies, no definitive conclusions has been drawn on the effectiveness of Bailing capsules in the treatment of lupus nephritis due to limited sample sizes and inconsistent results across these studies. 
The present study conducted a meta-analysis of studies which explored the use of Bailing capsules to treat lupus nephritis.

\section{Materials and methods}

Data source. Relevant clinical studies were obtained from PubMed (ncbi.nlm.nih.gov/pubmed/), Embase (embase.com), Cochrane Library (cochranelibrary.com/), China National Knowledge Infrastructure (cnki.net/), WanFang database (wanfangdata.com.cn/) and the Chinese Biomedical Literature Database (sinomed.ac.cn/). A literature search was performed using the following search strategy: Sources published between July 2004 and 2019 were searched, without language limitations, using the keywords including 'Bailing capsule', AND 'lupus nephritis' OR 'lupus erythematosus nephritis'.

Inclusion and exclusion criteria. The selected studies involved the use of Bailing capsules for the treatment of lupus nephritis. For repeated publications or when the same dataset was used for multiple studies, only the latest or the most comprehensive article was included. Reviews, reports, letters, comments and studies without complete data which could be used for statistical analysis were excluded.

Data extraction and quality assessment. The following data from each study: First author, publication year, study year, follow-up time, number of Bailing capsule and control (non-Bailing capsule) groups, medication, age, sex, course of the disease and outcome indicators including SLEDAI score, Alb levels, 24-h urinary protein, serum creatinine, anti-ds-DNAIg, C3 and the number of effective treatments and complications were extracted by two independent researchers. Quality assessment of the included studies was based on the guidelines recommended by the Cochrane Collaboration (15).

Statistical analysis. Meta-analysis was performed using R-3.12 software, and the odds ratio (OR) or standardized mean differences (SMD) and 95\% confidence interval (95\% CI) (16) were used for the effect index. Heterogeneity was analysed using the Cochran's Q test (17) and the $\mathrm{I}^{2}$ test. If $\mathrm{P}<0.05$ or $\mathrm{I}^{2}>50 \%$, indicating that all the studies were heterogeneous, the random effects model was applied. If not, the fixed effect model was used (18). Sensitivity analysis was conducted by trimming one study at a time (19). The pooled effect differences before and after the trim were compared. A reversal of the pooled results after the trim was indicative of unstable results. Publication bias was evaluated using Egger's test (20).

\section{Results}

Characteristics of the included studies. A total of 98 articles were obtained after excluding 49 duplicates. Then, 66 obvious irrelevant articles were eliminated. Two animal studies, six reviews, eight studies without useable data and two studies with duplicated populations were subsequently removed after abstract and full-text review. 14 articles $(11,13,14,21-31)$ were included in the present meta-analysis; the flow diagram of the search and selection process is presented in Fig. 1.

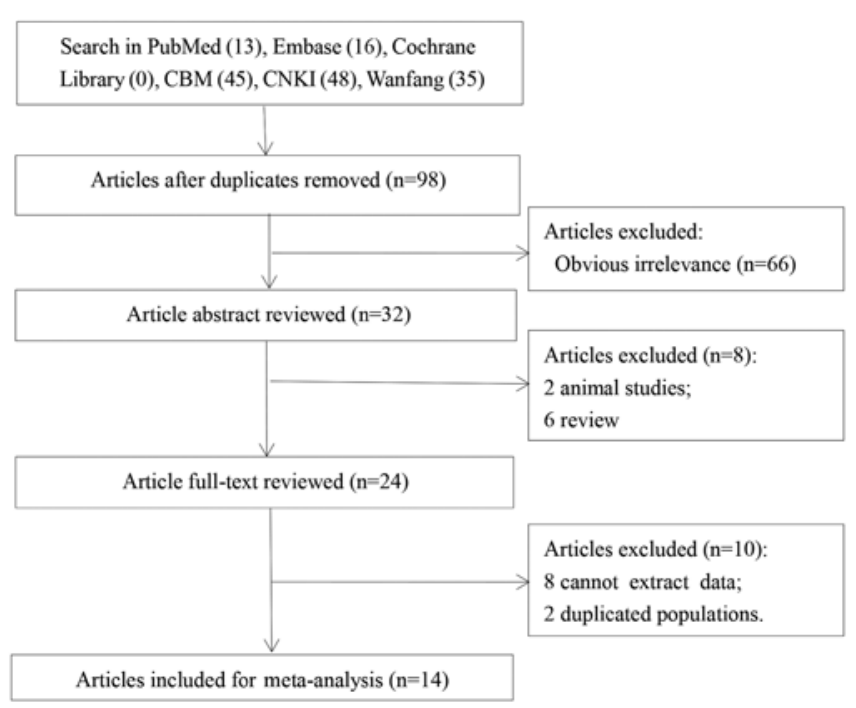

Figure 1. Flow diagram of the search and selection process. CBM, China Biology Medicine; CNKI, China National Knowledge Infrastructure.

The included studies were published between 2006 and 2019 and performed between 2000 and 2018. The follow-up time was 1-12 months. The studies comprised 1,301 participants, including 654 patients with lupus nephritis and 647 controls. The Bailing capsule group were treated with Bailing capsule combined with cortisone, prednisone, prednisolone, low molecular weight heparin, tacrolimus, methyprednisolone cyclophosphamide, methylprednisolone or leflunomide. No significant differences were observed in sex, age or course of the disease between the Bailing capsule and control groups in each publication (Table I). The outcome indicators, including SLEDAI score, complications (such as respiratory infection, fatigue, drowsiness, gastrointestinal symptoms, rash and leukocytopenia), Albumin, 24-h urinary protein, serum creatinine, $\mathrm{C} 3$ and the number of effective treatments and were recorded (Table II). The results of the quality assessment demonstrated high or unclear risk of bias (Fig. 2), indicating that the literature was of average quality.

Meta-analysis. The heterogeneity test demonstrated $\mathrm{P}<0.05$ and $\mathrm{I}^{2}>50 \%$ for SLEDAI score, Alb, 24-h urinary protein, complement $\mathrm{C} 3$ and serum creatinine. The random effects model was thus applied for these indicators. The fixed effect model was used for the other results.

Bailing capsule application was demonstrated to be more effective compared with treatment without the use of Bailing capsule for lupus nephritis (OR, 3.62; 95\% CI: 2.55, 5.13; $\mathrm{Z}=7.22 ; \mathrm{P}<0.001$; Fig. 3A). Sensitivity analysis demonstrated that the results were reliable; there was a publication bias for the effectiveness of treatment Egger's test, $\mathrm{t}=2.76 ; \mathrm{P}<0.001$ ).

No significant differences were observed in the anti-ds-DNAIg levels between the Bailing capsule and control groups (OR, $0.84 ; 95 \%$ CI: $0.56,1.27 ; \mathrm{Z}=0.83 ; \mathrm{P}=0.400$; Fig. 3B). Sensitivity analysis demonstrated that the results were reliable. There was a publication bias for anti-ds-DNAIg $(\mathrm{t}=33.40 ; \mathrm{P}=0.0001)$.

The number of complications in the Bailing Capsule group was lower compared with that in the control group (OR, 0.48; 95\% CI: 0.31, 0.75; Z=3.22; P=0.0013; Fig. 3C). Sensitivity 


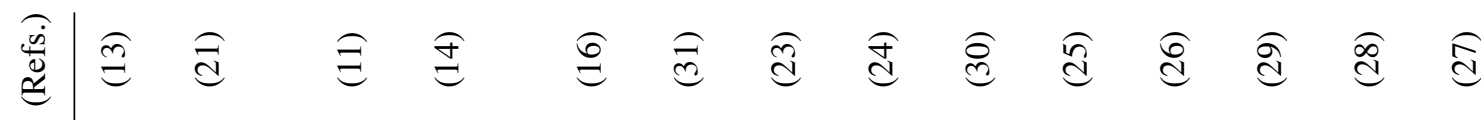

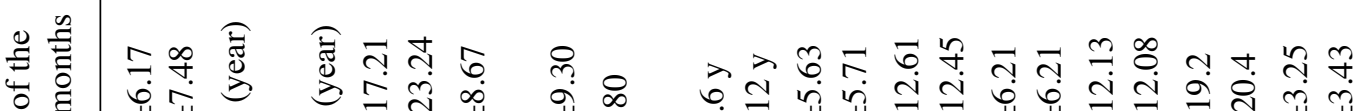

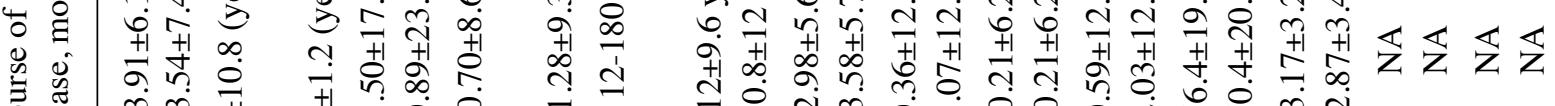

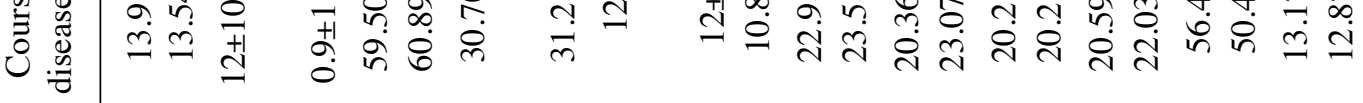

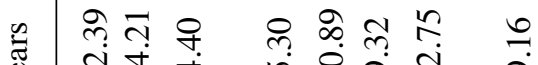

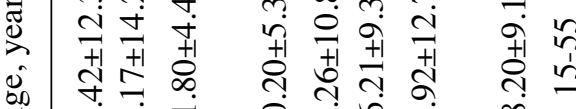

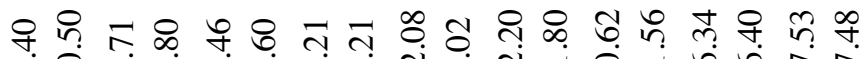

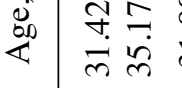

ñ

$\infty$

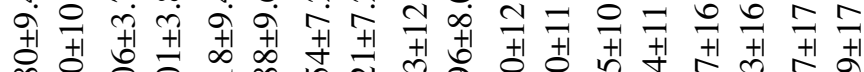

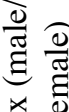

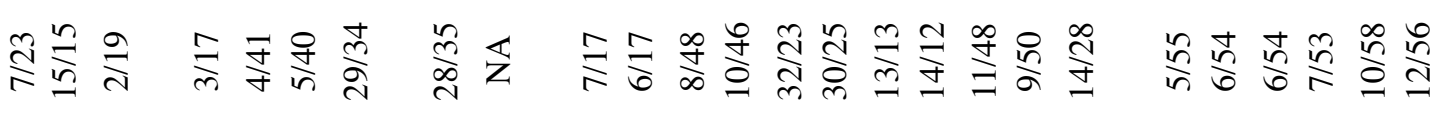

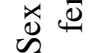

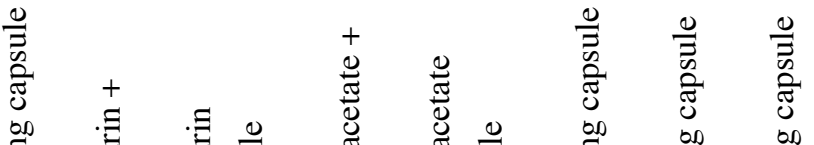

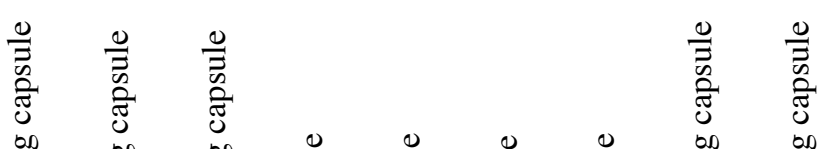

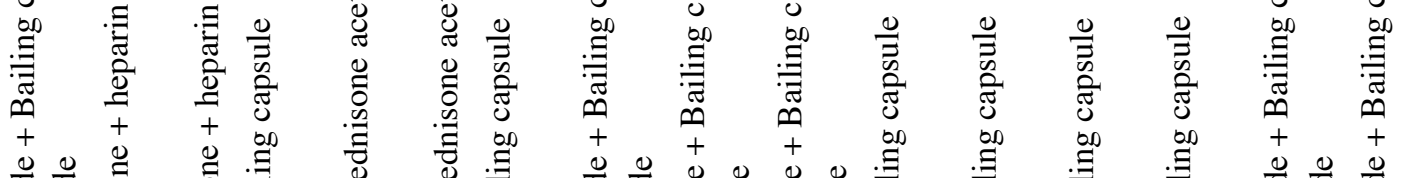

它

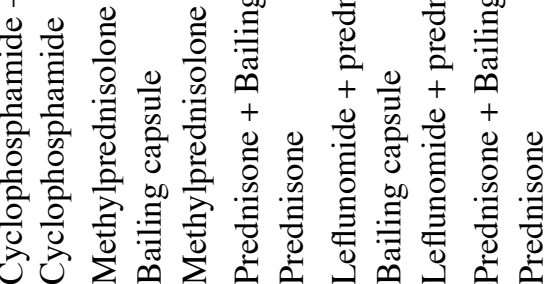

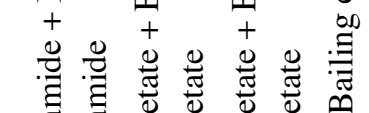

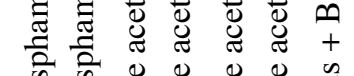

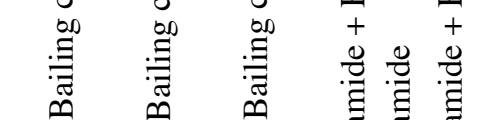

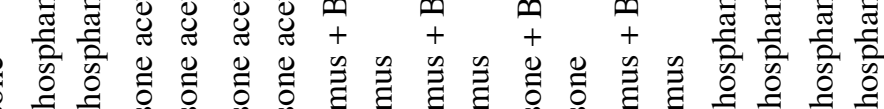

商 爷

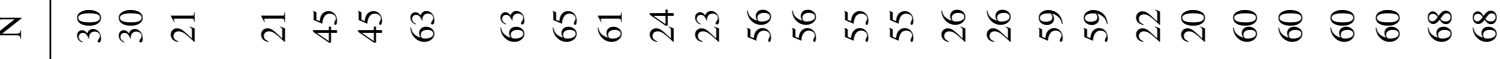

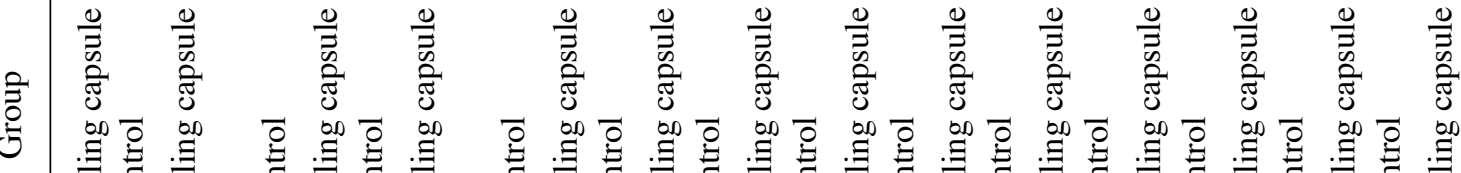

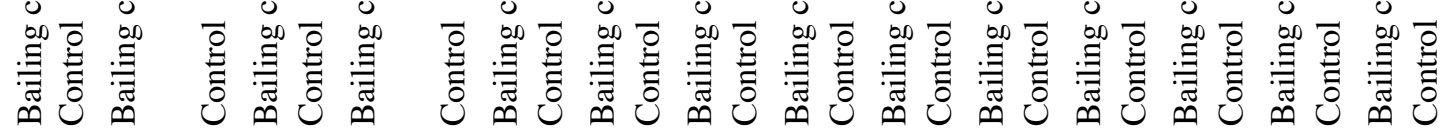

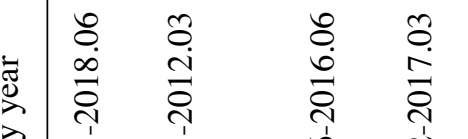

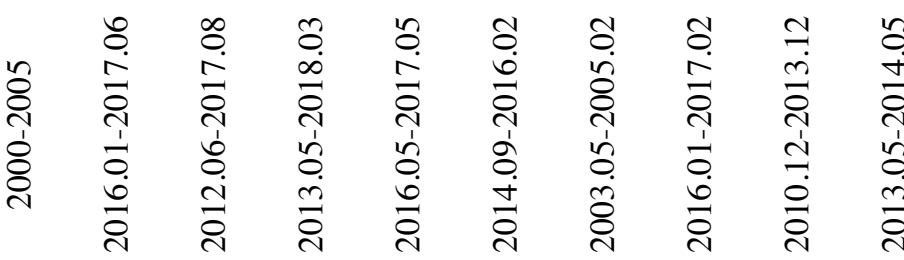

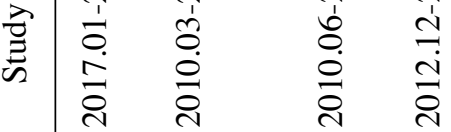

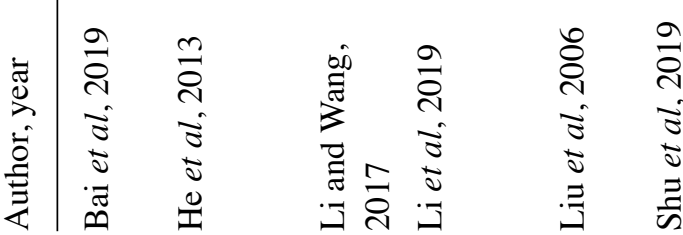

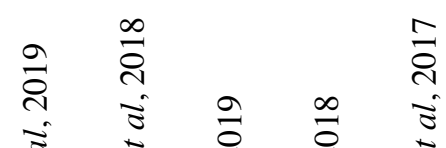

สิ

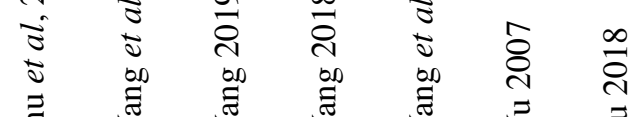




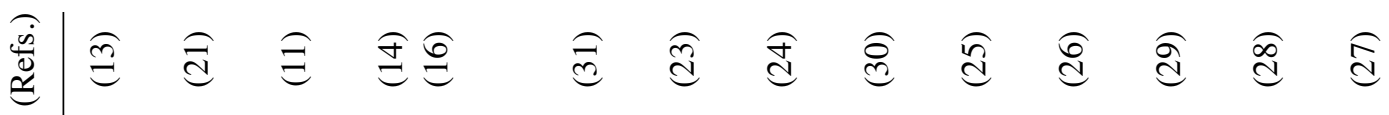

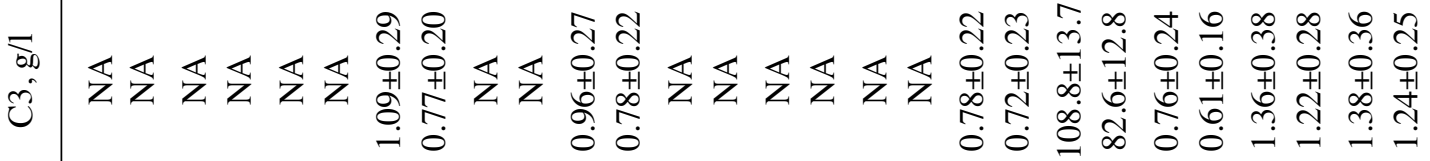

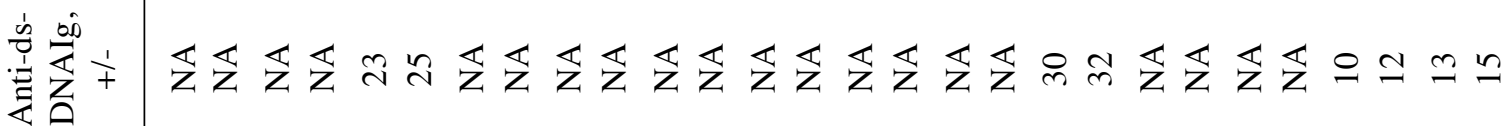

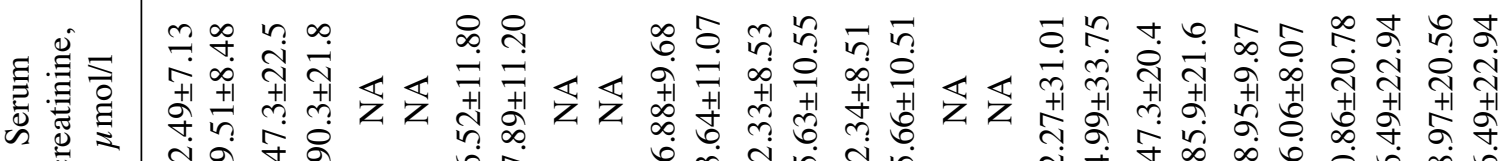

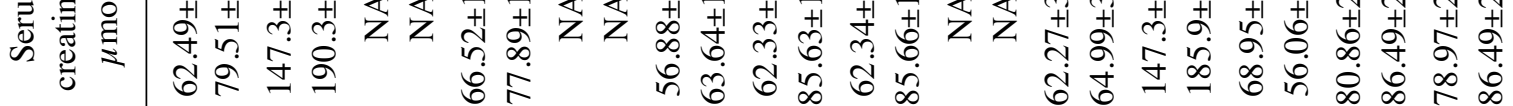

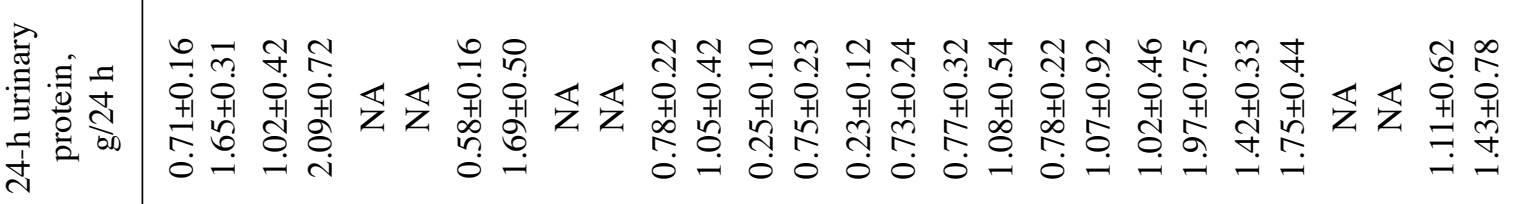

幽

$\sum_{0}^{\infty}$

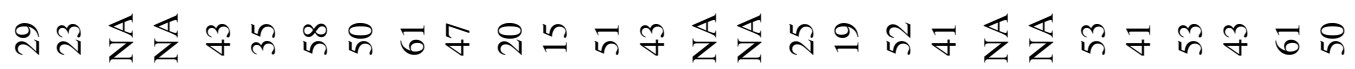

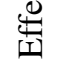

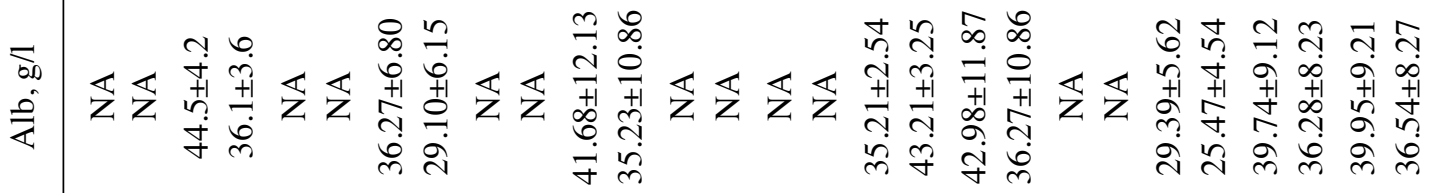
产

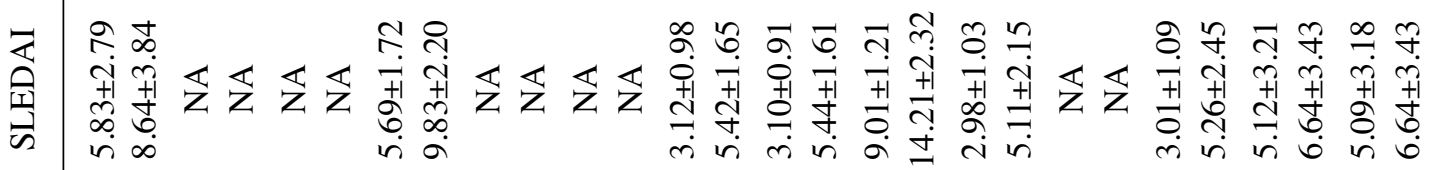

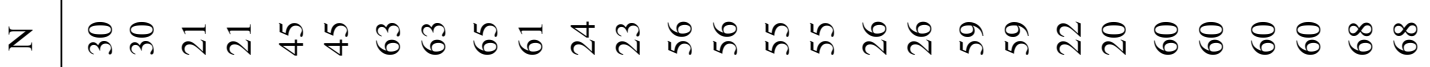

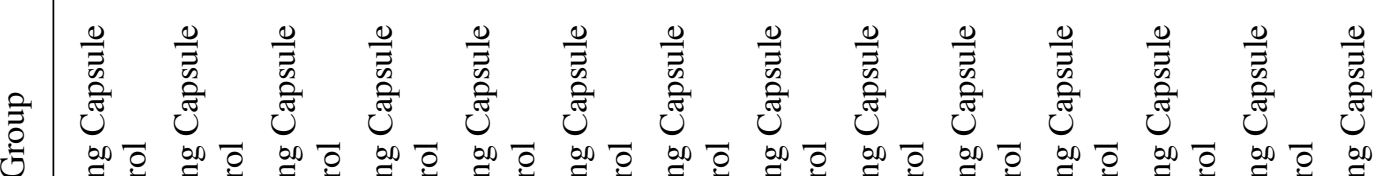
广

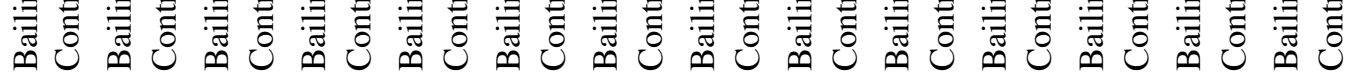

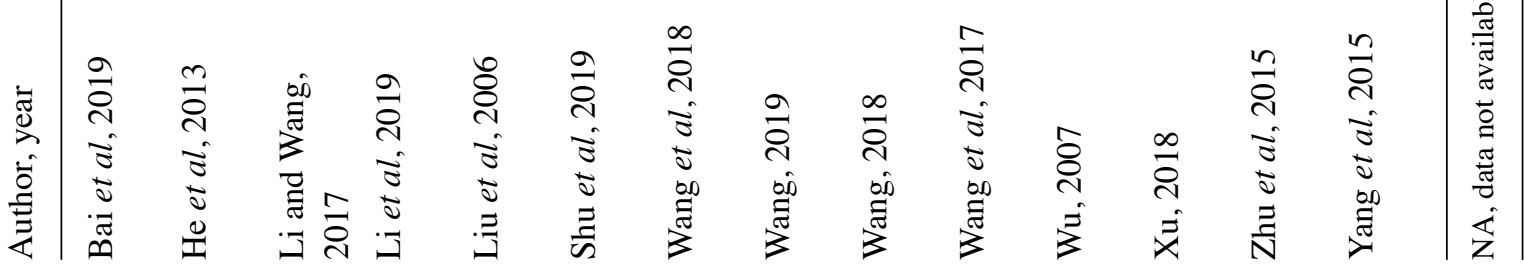


Random sequence generation (selection bias)

Allocation concealment (selection bias)

Blinding of participants and personnel (performance bias)

Blinding of outcome assessment (detection bias)

Incomplete outcome data (attrition bias)

Selective reporting (reporting bias)

Other bias
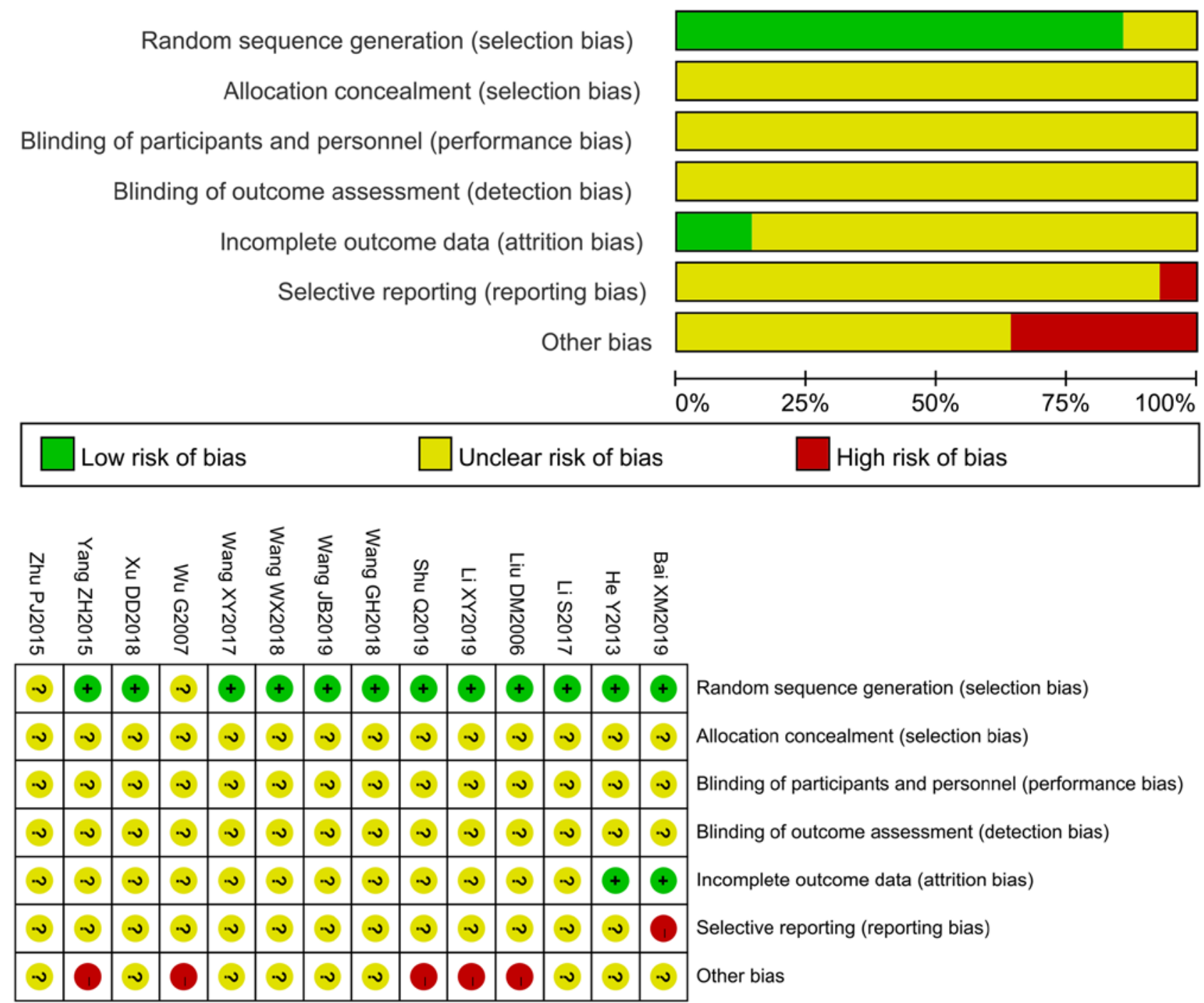

Figure 2. Quality assessment of the studies, based on the following criteria: Random sequence generation (selection bias), allocation concealment (selection bias), blinding of participants and personnel (performance bias), incomplete outcome data (attrition bias) and selective reporting (reporting bias).

analysis demonstrated that the results were reliable; there was no publication bias for complications ( $\mathrm{t}=0.45 ; \mathrm{P}=0.67)$.

The decrease in the SLEDAI score in the Bailing capsule group was higher compared with that in the control group (SMD, -1.35; 95\% CI: $-1.80,-0.90 ; \mathrm{Z}=5.89$; P<0.0001; Fig. 3D). Sensitivity analysis indicated that the results were reliable; no publication bias existed for the SLEDAI score $(\mathrm{t}=2.38 ; \mathrm{P}=0.05)$.

The decrease in 24-h urinary protein in the Bailing capsule group was greater compared with those in the control group (SMD, -1.67; 95\% CI: $-2.33,-1.01 ; \mathrm{Z}=5.89$; $\mathrm{P}<0.0001$; Fig. 4A). Sensitivity analysis demonstrated that the results were reliable and stable. Publication bias was identified for 24-h urinary protein $(\mathrm{t}=2.42 ; \mathrm{P}=0.04)$.

The decrease in serum creatinine in the Bailing capsule group was greater compared with those in the control group (SMD, -1.03; 95\% CI: -1.73, -0.33; $\mathrm{Z}=2.88, \mathrm{P}=0.004$; Fig. 4B). Sensitivity analysis suggested that the results were reliable and stable; there was no publication bias for serum creatinine $(\mathrm{t}=2.06 ; \mathrm{P}=0.07)$.

The increase in Alb in the Bailing capsule group was greater compared with those in the control group (SMD, 0.77;
95\% CI: 0.42, 1.12; $\mathrm{Z}=4.32 ; \mathrm{P}<0.0001 ;$ Fig. 4C). However, after eliminating the study of Wenxin W (30), the results of the pooled SMD were reversed. No publication bias was identified for Alb ( $\mathrm{t}=0.70 ; \mathrm{P}=0.51)$.

No significant differences were observed in the levels of complement $\mathrm{C} 3$ between the Bailing capsule and control groups (SMD, 0.42; 95\% CI: $-0.15,-0.99 ; \mathrm{Z}=1.45 ; \mathrm{P}=0.15$; Fig. 4D). Sensitivity analysis demonstrated that the results were reliable and stable; there was no publication bias for complement $\mathrm{C} 3(\mathrm{t}=1.73 ; \mathrm{P}=0.15)$.

\section{Discussion}

In this meta-analysis, a total of 14 studies comprising 1,301 participants were included to verify the effectiveness of Bailing capsules in the treatment of lupus nephritis. The results demonstrated that, with the exception of anti-ds-DNAIg and complement C3, other indicators (SLEDAI score, Alb, 24-h urinary protein, serum creatinine, and the number of effective treatments and complications) in the Bailing capsule group were improved compared with those in the control group. 
A Study

$\begin{array}{lllll}\text { Bai XM 2019 } & 29 & 30 & 23 & 30 \\ \text { Li S 2017 } & 43 & 45 & 35 & 45 \\ \text { Li XY 2019 } & 58 & 63 & 50 & 63 \\ \text { Liu DM 2006 } & 61 & 65 & 47 & 61 \\ \text { Shu Q 2019 } & 20 & 24 & 15 & 23 \\ \text { Wang GH 2018 } & 51 & 56 & 43 & 56 \\ \text { Wang WX 2018 } & 25 & 26 & 19 & 26 \\ \text { Wang XY 2017 } & 52 & 59 & 41 & 59 \\ \text { Xu DD 2018 } & 53 & 60 & 41 & 60 \\ \text { Zhu PJ 2015 } & 53 & 60 & 43 & 60 \\ \text { Yang ZH 2015 } & 61 & 68 & 50 & 68 \\ & & & & \\ \text { Fixed effect model } & & 556 & & 551 \\ \text { Random effects model } & & & & \\ \text { Heterogeneity: } I^{2}=0 \%, \tau^{2}=0, P=0.99 & & \end{array}$

Heterogeneity: $I^{2}=0 \%, \tau^{2}=0, P=0.99$

B

Study

Li S 2017

Wang XY 2017

Zhu PJ 2015

Yang ZH 2015

Fixed effect model

Experimental Control
Events Total Events Total

Experimental Control

Events Total Events Total

Random effects model

Heterogeneity: $I^{2}=0 \%, \tau^{2}=0, \mathrm{P}=1.00$

C

Study

Experimental Control

Events Total Events Total

Bai XM 2019

Liu DM 2006

Wang GH 2018

Wang JB 2019

Wang XY 2017

Xu DD 2018

Zhu PJ 2015

Yang ZH 2015

$\begin{array}{ll}23 & 4 \\ 30 & 5 \\ 10 & 6 \\ 13 & 68\end{array}$

232

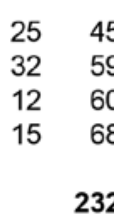

232

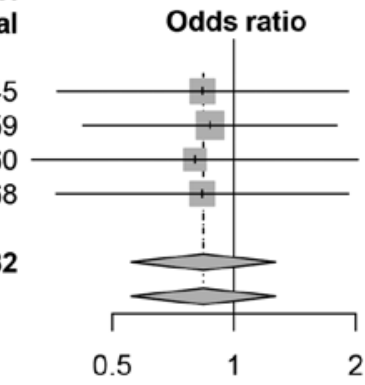

$\begin{array}{rrrr}3 & 30 & 11 & 30 \\ 37 & 65 & 52 & 61 \\ 6 & 56 & 4 & 56 \\ 4 & 55 & 6 & 55 \\ 16 & 59 & 28 & 59 \\ 15 & 60 & 28 & 60 \\ 23 & 60 & 22 & 60 \\ 18 & 68 & 30 & 68\end{array}$

Fixed effect model

453

Random effects model

Heterogeneity: $I^{2}=45 \%, \tau^{2}=0.1752, P=0.08$

D

Study

Bai XM 2019

Li XY 2019

Wang GH 2018

Wang JB 2019

Wang WX 2018

Wang XY 2017

Xu DD 2018

Zhu PJ 2015

Yang $\mathrm{ZH} 2015$

Fixed effect model

Random effects mode

Heterogeneity: $I^{2}=90 \%, \tau^{2}=0.4202, \mathrm{P}<0.01$
Experimental

$\begin{array}{cc}\text { Experimental } & \text { Control } \\ \text { Total Mean SD Total Mean SD }\end{array}$

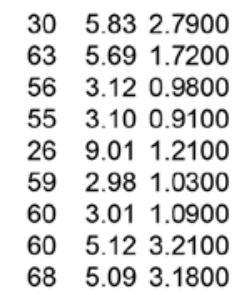

$30 \quad 8.64 \quad 3.8400$

$\begin{array}{lll}63 & 9.83 & 2.2000\end{array}$

$\begin{array}{lll}56 & 5.42 & 1.6500\end{array}$

$55 \quad 5.441 .6100$

$26 \quad 14.212 .3200$

$59 \quad 5.112 .1500$

$\begin{array}{lll}60 & 5.26 & 2.4500\end{array}$

$60 \quad 6.64 \quad 3.4300$

$\begin{array}{lll}68 & 6.64 & 3.4300\end{array}$

$\begin{array}{ll}477 & 477\end{array}$ $\begin{array}{lll}0.1 & 0.512 \quad 10\end{array}$

Odds ratio

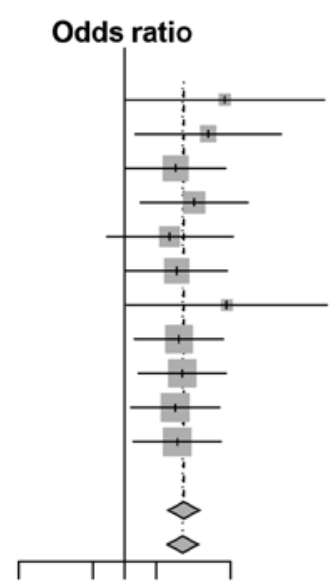

Weight Weight
(fixed) (random)

$8.83[1.01 ; 76.96] \quad 2.1 \% \quad 2.6 \%$

$6.14[1.26 ; 29.89] \quad 4.3 \% \quad 5.0 \%$

$3.02[1.01 ; 9.05] \quad 11.0 \% \quad 10.3 \%$

$4.54[1.40 ; 14.70] \quad 8.2 \% \quad 9.0 \%$

$2.67[0.67 ; 10.54] \quad 7.1 \% \quad 6.6 \%$

$3.08[1.02 ; 9.34] \quad 10.6 \% \quad 10.1 \%$

$9.21[1.04 ; 81.36] \quad 2.0 \% \quad 2.6 \%$

$3.26[1.24 ; 8.55] \quad 13.4 \% \quad 13.3 \%$

$3.51 \quad[1.35 ; 9.14] \quad 13.2 \% \quad 13.5 \%$

$2.99[1.14 ; 7.88] \quad 13.9 \% \quad 13.2 \%$

$3.14[1.21 ; 8.11] \quad 14.2 \% \quad 13.8 \%$

$3.62[2.55 ; 5.13] 100.0 \%$

$3.54[2.49 ; 5.03] \quad--\quad 100.0 \%$

Weight Weight

OR $\quad 95 \%-\mathrm{Cl}$ (fixed) (random)

$0.84[0.37 ; 1.92] \quad 24.4 \% \quad 24.4 \%$

$0.87[0.42 ; 1.80] \quad 31.4 \% \quad 32.0 \%$

$0.80[0.32 ; 2.02] \quad 20.0 \% \quad 19.5 \%$

$0.84[0.36 ; 1.92] \quad 24.2 \% \quad 24.1 \%$

$0.84[0.56 ; 1.27] 100.0 \%$ $0.84[0.56 ; 1.27] \quad-\quad 100.0 \%$

Weight Weight

OR $\quad 95 \%-\mathrm{Cl}$ (fixed) (random)

$0.19[0.05 ; 0.78] \quad 8.3 \% \quad 7.3 \%$

$0.23[0.10 ; 0.54] \quad 19.4 \% \quad 13.7 \%$

$1.56[0.42 ; 5.86] \quad 3.0 \% \quad 8.0 \%$

$0.64[0.17 ; 2.41] \quad 4.7 \% \quad 8.0 \%$

$0.41[0.19 ; 0.89] \quad 17.1 \% \quad 15.4 \%$

$0.38[0.18 ; 0.83] \quad 17.6 \% \quad 15.3 \%$

$1.07[0.51 ; 2.25] \quad 11.4 \% \quad 15.9 \%$

$0.46[0.22 ; 0.94] \quad 18.5 \% \quad 16.3 \%$

$\begin{array}{llr}0.48[0.35 ; 0.65] & 100.0 \% & -- \\ 0.48[0.31 ; 0.75] & -- & 100.0 \%\end{array}$

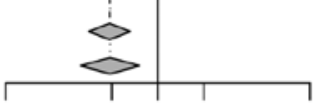

$\begin{array}{lllll}0.1 & 0.5 & 1 & 2 & 10\end{array}$

Standardised mean difference

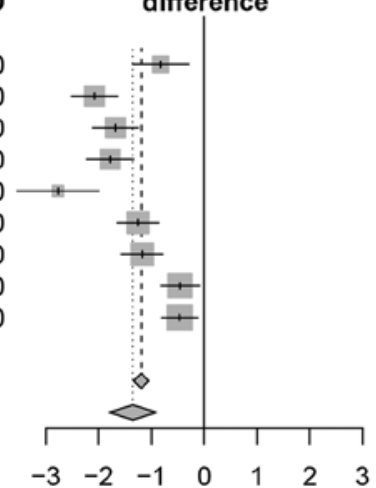

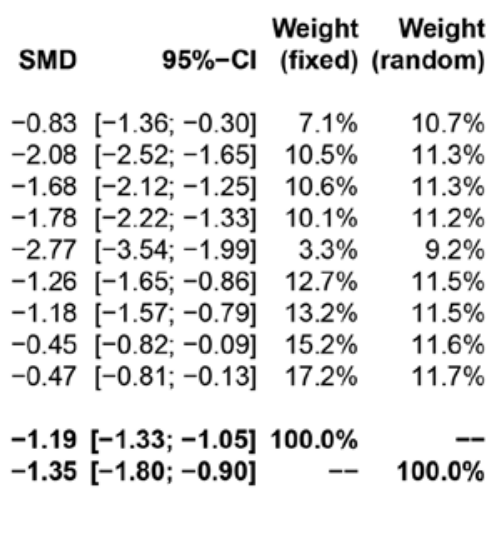

Figure 3. Forest plot of meta-analysis results of (A) effectiveness, (B) anti-ds-DNAIg, (C) complication (D) and SLEDAI score. SLEDAI, systemic lupus erythematosus disease activity index; OR, odds ratio; CI, confidence interval.

Quantitative analysis of the 24-h urinary protein levels is an important diagnostic index to assess the severity and prognosis of nephritis or kidney-related diseases (32). The SLEDAI score is an index used to assess SLE disease activity (33). Serum creatinine is positively associated with the degree of early renal injury in patients with acute glomerulonephritis (33). The 
A Study

Bai XM 2019

He Y 2013

Li XY 2019

Shu Q 2019

Wang GH 2018

Wang JB 2019

Wang WX 2018

Wang XY 2017

Wu G 2007

Xu DD 2018

Yang ZH 2015

Fixed effect model

Random effects model

Heterogeneity: $I^{2}=95 \%, \tau^{2}=1.1597, \mathrm{P}<0.01$

B

Study

Bai XM 2019

$\mathrm{He}$ Y 2013

Li XY 2019

Shu Q 2019

Wang GH 2018

Wang JB 2019

Wang XY 2017

Wu G 2007

Xu DD 2018

Zhu PJ 2015

Yang ZH 2015

Fixed effect model

Random effects model

Heterogeneity: $I^{2}=96 \%, \tau^{2}=1.3490, P<0.01$

C

Study

He Y 2013
Li XY 2019
Shu Q 2019
Wang WX 2018
Wang XY 2017
Xu DD 2018
Zhu PJ 2015
Yang ZH 2015

Experimental

Experimental Total Mean Contro

$\begin{array}{lll}30 & 62.49 & 7.1300\end{array}$ $\begin{array}{lll}21 & 147.30 & 22.5000\end{array}$

$\begin{array}{lll}63 & 66.52 & 11.8000\end{array}$

$\begin{array}{lll}24 & 56.88 & 9.6800\end{array}$

$\begin{array}{lll}56 & 62.33 & 8.5300\end{array}$

$\begin{array}{lll}55 & 62.34 & 8.5100\end{array}$

$\begin{array}{lll}59 & 62.27 & 31.0100\end{array}$

22147.3020 .4000

$\begin{array}{lll}60 & 68.95 \quad 9.8700\end{array}$

$\begin{array}{lll}60 & 80.86 & 20.7800\end{array}$

$\begin{array}{llll}68 & 78.97 & 20.5600\end{array}$

518

515

Fixed effect model

Random effects model

Heterogeneity: $I^{2}=93 \%, \tau^{2}=0.6182, \mathrm{P}<0.01$

D

Study

Li XY 2019

Shu Q 2019

Wang XY 2017

Wu G 2007

Xu DD 2018

Zhu PJ 2015

Yang ZH 2015

Fixed effect model

Random effects model

Heterogeneity: $I^{2}=79 \%, \tau^{2}=0.1690, P<0.01$

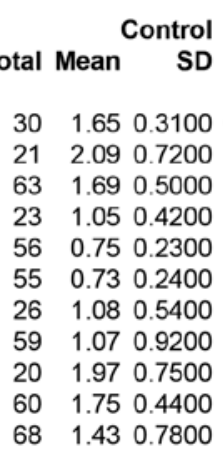

481

Control SD
SD

$\begin{array}{llllll}21 & 44.50 & 4.2000 & 21 & 36.10 & 3.6000\end{array}$ $\begin{array}{llllll}63 & 36.27 & 6.8000 & 63 & 29.10 & 6.1500\end{array}$

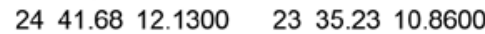
$\begin{array}{llllll}26 & 35.21 & 2.5400 & 26 & 43.21 & 3.2500\end{array}$ $\begin{array}{llllll}59 & 42.98 & 11.8700 & 59 & 36.27 & 10.8600\end{array}$ $\begin{array}{llllll}60 & 29.39 & 5.6200 & 60 & 25.47 & 4.5400\end{array}$ $\begin{array}{lllllll}60 & 39.74 & 9.1200 & 60 & 36.28 & 8.2300\end{array}$ $\begin{array}{llllll}68 & 39.95 & 9.2100 & 68 & 36.54 & 8.2700\end{array}$

381

380

$\begin{array}{lll}59 & 0.78 & 0.2200\end{array}$

356

353

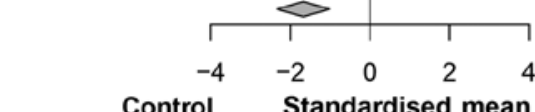
Total Mean SD $\begin{array}{r}\text { Control } \\ \text { difference }\end{array}$

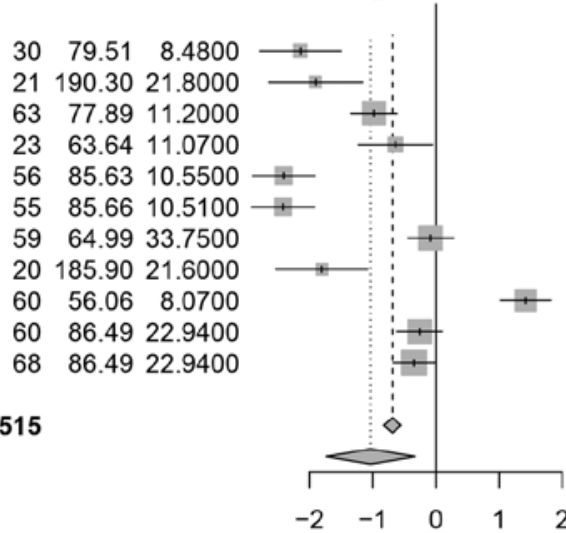

Standardised mean difference
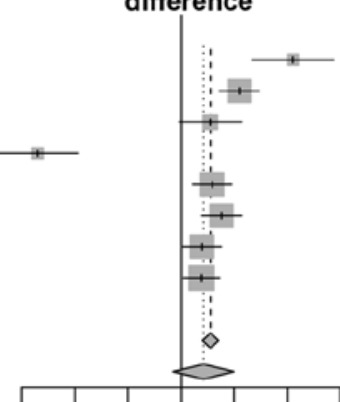

$\begin{array}{lllllll}-3 & -2 & -1 & 0 & 1 & 2 & 3\end{array}$

Control Standardised mean Total Mean SD Total Mean SD

$\begin{array}{llllll}63 & 1.09 & 0.2900 & 63 & 0.77 & 0.2000\end{array}$

$\begin{array}{llllll}24 & 0.96 & 0.2700 & 23 & 0.78 & 0.2200\end{array}$

$22108.8013 .7000 \quad 2082.6012 .8000$

$\begin{array}{llllll}60 & 0.76 & 0.2400 & 60 & 0.61 & 0.1600\end{array}$

$\begin{array}{llllll}60 & 1.36 & 0.3800 & 60 & 1.22 & 0.2800\end{array}$

$\begin{array}{llllll}68 & 1.38 & 0.3600 & 68 & 1.24 & 0.2500\end{array}$

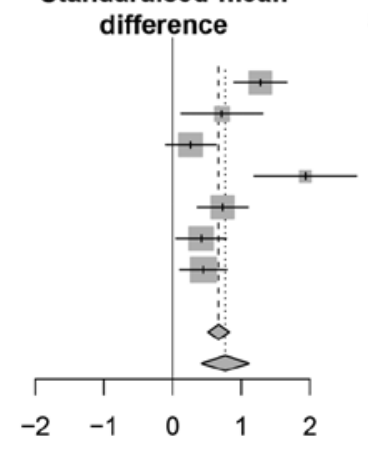

SMD Weight Weight

(fixed) (random)

$-3.76[-4.62 ;-2.90] \quad 2.9 \% \quad 8.4 \%$

$-1.78[-2.51 ;-1.06] \quad 4.1 \% \quad 8.7 \%$

$-2.97[-3.48 ;-2.46] \quad 8.4 \% \quad 9.2 \%$

$-0.80[-1.39 ;-0.20] \quad 6.1 \% \quad 9.0 \%$

$-2.80[-3.33 ;-2.27] \quad 7.9 \% \quad 9.2 \%$

$-2.62[-3.13 ;-2.10] \quad 8.3 \% \quad 9.2 \%$

$-0.69[-1.25 ;-0.13] \quad 6.9 \% \quad 9.1 \%$

$-0.43[-0.80 ;-0.07] \quad 16.4 \% \quad 9.5 \%$

$-1.52[-2.21 ;-0.82] \quad 4.5 \% \quad 8.8 \%$

$-0.84[-1.22 ;-0.47] \quad 15.6 \% \quad 9.5 \%$

$-0.45[-0.79 ;-0.11] \quad 18.8 \% \quad 9.5 \%$

$\begin{array}{lrr}-1.32[-1.47 ;-1.18] & 100.0 \% & -- \\ -1.67[-2.33 ;-1.01] & - & 100.0 \%\end{array}$

Weight Weight

SMD $\quad 95 \%-\mathrm{Cl}$ (fixed) (random)

$-2.14[-2.79 ;-1.50] \quad 4.4 \% \quad 8.9 \%$

$-1.90[-2.65 ;-1.16] \quad 3.3 \% \quad 8.6 \%$

$-0.98[-1.35 ;-0.61] \quad 13.3 \% \quad 9.3 \%$

$-0.64[-1.23 ;-0.05] \quad 5.3 \% \quad 9.0 \%$

$-2.41[-2.90 ;-1.92] \quad 7.6 \% \quad 9.1 \%$

$-2.42[-2.92 ;-1.93] \quad 7.4 \% \quad 9.1 \%$

$-0.08[-0.44 ; 0.28] \quad 14.0 \% \quad 9.3 \%$

$-1.81[-2.53 ;-1.08] \quad 3.4 \% \quad 8.7 \%$

$1.42 \quad[1.02 ; 1.82] \quad 11.3 \% \quad 9.3 \%$

$-0.26[-0.61 ; 0.10] \quad 14.1 \% \quad 9.3 \%$

$-0.34[-0.68 ; 0.00] \quad 15.9 \% \quad 9.4 \%$

$\begin{array}{rrr}-0.69[-0.82 ;-0.55] & 100.0 \% & -- \\ -1.03[-1.74 ;-0.33] & -- & 100.0 \%\end{array}$

Weight Weight

95\%-Cl (fixed) (random)

$2.11[1.34 ; 2.88] \quad 3.8 \% \quad 11.1 \%$

$1.10 \quad[0.72 ; 1.47] \quad 16.0 \% \quad 13.1 \%$

$0.55[-0.03 ; 1.13] \quad 6.6 \% \quad 12.1 \%$

$-2.70[-3.47 ;-1.94] \quad 3.8 \% \quad 11.1 \%$

$0.59 \quad[0.22 ; 0.95] \quad 16.6 \% \quad 13.1 \%$

$0.76 \quad[0.39 ; 1.13] \quad 16.4 \% \quad 13.1 \%$

$0.40 \quad[0.03 ; 0.76] \quad 17.2 \% \quad 13.1 \%$

$0.39[0.05 ; 0.73] \quad 19.6 \% \quad 13.2 \%$

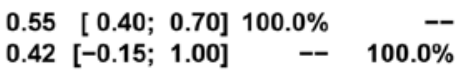

Weight Weight

1.28 [ $0.89 ; 1.66] \quad 15.9 \% \quad 15.3 \%$

$0.72[0.13 ; 1.31] \quad 6.7 \% \quad 12.2 \%$

$0.26[-0.10 ; 0.63] \quad 17.9 \% \quad 15.6 \%$

$1.94[1.19 ; 2.68] \quad 4.2 \% \quad 10.1 \%$

$0.73[0.36 ; 1.10] \quad 17.1 \% \quad 15.5 \%$

$0.42[0.05 ; 0.78] \quad 17.9 \% \quad 15.6 \%$

$0.45[0.11 ; 0.79] \quad 20.2 \% \quad 15.9 \%$

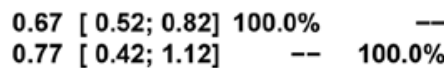

Figure 4. Forest plot of meta-analysis results of (A) 24-h urinary protein, (B) serum creatinine, (C) Alb (D) and complement C3. Alb, albumin; SMD, standardised mean difference; $\mathrm{CI}$, confidence interval.

level of Alb, which reflects the nutritional status of patients, is associated with lupus nephritis development, and nephrotic syndrome is often accompanied by hypoalbuminemia (34). A significant reduction in complement $\mathrm{C} 3$, which is produced by liver cells, is related to the occurrence of SLE and other immune diseases, and anti-ds-DNAIg is the main immune factor 
involved in the organ damage in SLE (25). In a meta-analysis by Wang et al (35), several indicators including serum creatinine, complement C3, 24-h urinary protein, adverse effects and SLEDAI score were improved in the Bailing capsule group compared with those in the non-Bailing capsule group for lupus nephritis therapy. In the present meta-analysis, SLEDAI score, Alb, 24-h urinary protein, serum creatinine, and the number of effective treatments and complications in the Bailing capsule group were improved compared with those in the control group. Thus, Bailing capsules may be used effectively in the treatment of lupus nephritis.

However, the present study has certain limitations; there was significant heterogeneity among the studies, likely caused by differences in habits and customs, living conditions and economic development level in different regions. In addition, the effects of other confounding factors, such as sex and age, may have contributed to this heterogeneity. In addition, due to incomplete data, correction of covariates and subgroup analysis were not performed. The number of eligible studies was further reduced due to the rigorous exclusion and inclusion criteria (36). Additionally, all the included studies were from China, which may have caused a selection bias. Publication bias was also observed for effectiveness, anti-ds-DNAIg and 24-h urinary protein. Furthermore, in the sensitivity analysis of Alb, after eliminating the study of Wang (30), the results of the pooled SMD were reversed, indicating an unreliable outcome. Lastly, since Bailing capsule was used for SLE or lupus nephritis treatment in combination with other medicines, these medicines were different in different studies, and the numbers of eligible studies for each medicine were low; thus, meta-analysis data to demonstrate the effect to Bailing Capsule therapy with other medicines was not added. Despite these limitations, the present study suggests that Bailing Capsules may be used effectively in lupus nephritis treatment. An updated meta-analysis drawing from larger scale studies and high-quality data may validate the findings of the present study.

\section{Acknowledgements}

Not applicable.

\section{Funding}

The present study was supported by the National Natural Science Foundation of China (grant no. 81770766) and Provincial Natural Science Foundation of Liaoning (grant no. 20170540999).

\section{Availability of data and materials}

The datasets used and/or analyzed during the current study are available from the corresponding author on reasonable request.

\section{Authors' contributions}

YL, TX and LY conceptualized and designed the study. YL, TX, XQ, BT and CB drafted and revised the manuscript. YL and TX performed the literature search, retrieved data and wrote the draft for the final manuscript. XQ performed statistical analysis. BT and CB plotted the tables and figures. YL supervised the project and approved the manuscript to be published. All authors read and approved the final manuscript.

\section{Ethics approval and consent to participate}

Not applicable.

\section{Patient consent for publication}

Not applicable.

\section{Competing interests}

The authors declare that they have no competing interests.

\section{References}

1. Sun L, Zou LX, Han YC, Wu L, Chen T, Zhu DD and Hu P: A20 overexpression exerts protective effects on podocyte injury in lupus nephritis by downregulating UCH-L1. J Cell Physiol 234: 16191-16204, 2019.

2. Mu Q, Zhang H, Liao X, Lin K, Liu H, Edwards MR, Ahmed SA, Yuan R, Li L, Cecere TE, et al: Control of lupus nephritis by changes of gut microbiota. Microbiome 5: 73, 2017.

3. Miranda-Hernández D, Cruz-Reyes C, Angeles U, Jara LJ and Saavedra MA: Prognostic factors for treatment response in patients with lupus nephritis. Reumatol Clin 10: 164-169, 2014 (In English, Spanish).

4. Rees F, Doherty M, Grainge MJ, Lanyon P and Zhang W: The worldwide incidence and prevalence of systemic lupus erythematosus: A systematic review of epidemiological studies. Rheumatology (Oxford) 56: 1945-1961, 2017.

5. Hogan J and Appel GB: Update on the treatment of lupus nephritis. Curr Opin Nephrol Hypertens 22: 224-230, 2013.

6. Okpechi IG, Gcelu A and Ameh OI: Lupus nephritis: A simplified approach to diagnosis and treatment in South Africa. South African Medical J 105: 1071-1074, 2015.

7. Chan TM: Treatment of severe lupus nephritis: The new horizon. Nat Rev Nephrol 11: 46-61, 2015.

8. Malvar A, Pirruccio P, Alberton V, Lococo B, Recalde C, Fazini B, Nagaraja H, Indrakanti D and Rovin BH: Histologic versus clinical remission in proliferative lupus nephritis. Nephrol Dial Transplant 32: 1338-1344, 2015.

9. Ren HJ, Sun YL and Yuan B: Chinese patent medicine Bailing capsule for treating lupus nephritis: A protocol for systematic review and meta-analysis. Medicine (Baltimore) 98: e17041, 2019.

10. Xu H and Li S: Pharmacological effects of Bailing capsule and its application in lung disease research. Zhongguo Zhong Yao Za Zhi 35: 2777-2781, 2010 (In Chinese).

11. Li S and Wang L: Clinical observation on the therapeutic effect of Corbrin Capsule in the treatment of lupus nephritis. Chin Commun Doctors 33: 61-62, 2017 (In Chinese).

12. Zhou L, Zhang B, Yao C and Liao A: Clinical research of Bailing capsules on change of cellular immunity in lupus nephritis. Chin Pharmacist (Issue 4): 289-290, 2004 (In Chinese).

13. Bai XM, Li H, Li XD and Li Y: Clinical study on corbrin capsules combined with cyclophosphamide in treatment of lupus nephritis. Drugs Clin 34: 1181-1184, 2019 (In Chinese).

14. Li XY, Li FX and Li B: Clinical study on corbrin capsules combined with leflunomide and prednisone in treatment of lupus nephritis. Drugs Clin 34: 154-158, 2019 (In Chinese).

15. Higgins JP and Green S (eds): Cochrane handbook for systematic reviews of interventions: Cochrane Book Series. The Cochrane Collaboration, 2008.

16. Liu T, Xu QE, Zhang $\mathrm{CH}$ and Zhang P: Occupational exposure to methylene chloride and risk of cancer: A meta-analysis. Cancer Causes Control 24: 2037-2049, 2013.

17. Lau J, Ioannidis JP and Schmid CH: Quantitative synthesis in systematic reviews. Ann Intern Med 127: 820-826, 1997.

18. Feng RN, Zhao C, Sun CH and Li Y: Meta-analysis of TNF 308 G/A polymorphism and type 2 diabetes mellitus. PLoS One 6: e18480, 2011 . 
19. Ma E, Wang H, Guo J, Tian R and Wei L: The association between the rs11196218A/G polymorphism of the TCF7L2 gene and type 2 diabetes in the Chinese Han population: A meta-analysis. Clinics (Sao Paulo) 70: 593-599, 2015.

20. Seagroatt V and Stratton I: Bias in meta-analysis detected by a simple, graphical test. Test had $10 \%$ false positive rate. BMJ 316 : 469-471, 1997.

21. He Y, Huang JP, Wu RY and Tan F: Observation of clinical effect of Bailing capsule combined with low molecular weight heparin for the treatment of lupus of nephritis. Asia Pacific Tradit Med 9: 207-208, 2013 (In Chinese).

22. Liu DM, Wang CH and Li XP: Clinical analysis of 65 cases of lupus nephritis treated by Bailing capsule. Chin Commun Doctors: 21-21, 2006 (In Chinese)

23. Wang GH, Su XD and Wu Y: Clinical study on Corbrin Capsules combined with prednisone in treatment of lupus nephritis. Drugs Clin 33: 2372-2376, 2018 (In Chinese)

24. Wang JB: Clinical effect of boling capsule combined with prednisone in the treatment of lupus nephritis SLEDAI scoring analysis. Chin J Mod Drug Appl: 121-122, 2019 (In Chinese)

25. Wang XY, Wang GJ, Zhang XX, Gong YN, Li YS, Ma S, Xiao J and Zhao ZZ: Clinical study on Bailing capsules combined with tacrolimus in treatment of lupus nephritis. Drugs Clin 32: 1065-1069, 2017 (In Chinese).

26. Wu G: Methyprednisolone with Bailing capsule in treatment of lupus nephritis. J Medical Forum 28: 38-39, 2007 (In Chinese).

27. Yang ZH, Liu B, Lu J, Hou XX and Yin J: A multicenter prospective study of the effect of Bailing capsules on clinical efficacy and infection rate in the patients with lupus nephritis. Heilongjiang Med J 28: 1205-1208, 2015 (In Chinese).
28. Zhu PJ, Ke SS and Xu F: Effect of Bailing capsules on interleukin-2, complement and infection rate in patients with lupus nephritis. Chin J Mod Appl Pharm 33: 364-368, 2015 (In Chinese).

29. Xu DD: Observation on the efficacy of boling capsule combined with tacrolimus in the treatment of lupus nephritis. Mod Diagn Treat 29: 2888-2890, 2018.

30. Wang WX: Study on the combination of boling capsule and tacrolimus in the treatment of lupus nephritis. Chin J Mod Drug Appl 12: 112-113, 2018.

31. Shu Q, Tan F, Huang LH and Peng J: Effect of Bailing capsules on macrophage function by regulating miR- 127 expression in lupus nephritis patients. Tradit Chinese Drug Res Clin Pharmacol 30: 733-738, 2019 (In Chinese)

32. Feng D: The correlation analysis of urine microalbumin /urine creatinine and $24 \mathrm{~h}$ urine proteinquantification in patients with chronic nephritis. Laborat Med Clin 14: 194, 2017 (In Chinese).

33. Zhang $\mathrm{H}$ and Ge X: Significance of Cys C, BUN and sCr level detection in assessment of early renal damage of acute glomerulonephritis. J Hainan Med Univ 22: 447-449, 2016 (In Chinese).

34. Gladman DD, Ibañez D and Urowitz MB: Systemic lupus erythematosus disease activity index 2000. J Rheumatol 29: 288-291, 2002.

35. Wang BL, Li QY and Huang WL: Assessment of renal injury degree in patients with nephritis by $\beta 2-\mathrm{m}, \mathrm{AIb}, \mathrm{IgG}$, and $\alpha 1-\mathrm{m}$. J Radioimmunol 14: 91-93, 2001.

36. Szulińska M, Skrypnik D, Ratajczak M, Karolkiewicz J, Madry E, Musialik K, Walkowiak J, Jakubowski $\mathrm{H}$ and Bogdański P: Effects of endurance and endurance-strength exercise on renal function in abdominally obese women with renal hyperfiltration: A prospective randomized trial. Biomed Environ Sci 29: 706-712, 2016 